\title{
Dynamics of AC susceptibility and coercivity behavior in nanocrystalline $\mathrm{TbAl}_{1.5} \mathrm{Fe}_{0.5}$ alloys
}

\author{
D. P. Rojas ${ }^{a, *}$ L. Fernández Barquín ${ }^{b}$, L. González Legarreta ${ }^{\mathrm{b}}$, J. Chaboy ${ }^{\mathrm{c}}$, C. Piquer ${ }^{\mathrm{c}}$, I. de Pedro ${ }^{\mathrm{b}}$, \\ J. Rodríguez Fernández ${ }^{\mathrm{b}}$ \\ ${ }^{a}$ Departamento de Física e Instalaciones-ETSAM, Universidad Politécnica de Madrid, Av. Juan Herrera, 4. 28040, Madrid. Spain

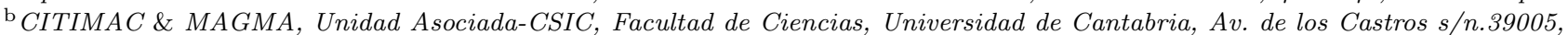 \\ Santander. Spain \\ ${ }^{\mathrm{c}}$ ICMA and Departamento de Física de la Materia Condensada, CSIC-Universidad de Zaragoza, 50009, Zaragoza. Spain
}

\begin{abstract}
The static and dynamic magnetic macroscopic properties of bulk and nanocrystalline $\mathrm{TbAl}_{1.5} \mathrm{Fe}_{0.5}$ alloys have been investigated. In bulk state, this alloy is understood as a reentrant ferromagnet. This is characterized by a ferromagnetic Curie transition at $114 \mathrm{~K}$, as deduced from magnetization including Arrott plots, higher than that of $\mathrm{TbAl}_{2}$. The reentrance is found at lower temperatures, below $54 \mathrm{~K}$, with a cluster glass behavior setting in, deduced from the magnetization irreversibility. This is accompanied by an abrupt increase of the coercivity from $0.08 \mathrm{kOe}$ to $15 \mathrm{kOe}$ at $5 \mathrm{~K}$, respect to the $\mathrm{TbAl}_{2}$ alloy. Room temperature Mössbauer spectroscopy confirms the paramagnetic state of such a bulk alloy. The spin dynamics within the disordered magnetic state is described by the AC-susceptibility which shows a Vogel-Fulcher law for the slowing down process. This is caused by a random anisotropy affecting the existing clusters. The production of milled $\mathrm{TbAl}_{1.5} \mathrm{Fe}_{0.5}$ alloys enhances the presence of magnetic disorder and results in the particle downsizing toward the nanocrystalline state (close to $10 \mathrm{~nm}$ ). In this case, two frequency-dependent contributions exist, with different activation energies, one of them can not be described by ideal spin glass nor blocking/unblocking (nanoparticle) processes. In addition, the coercivity reduces to $1 \mathrm{kOe}$ with the decrease of the size as a consequence of the existence of single domain particles. The results are explained by the intricate interplay between exchange interactions and magnetocrystalline anisotropy with disorder and size effects.
\end{abstract}

Key words: Tb alloys; nanocrystalline; Coercivity; AC-susceptibility

\section{Introduction}

Rare-earth (Re) magnetic materials have constituted a subject of exhaustive studies because their potential applications as permanent magnets [1,2]. Very recently, the study of size effects as a function of the milling conditions on $\mathrm{SmCo}_{5}, \mathrm{PrCo}_{5}$ and $\mathrm{Sm}_{2}(\mathrm{Co}, \mathrm{Fe})_{17}$ nanoparticles, obtained by surfactant-assisted ball milling, has been reported. By milling nanocrystalline precursor alloys, $\mathrm{SmCo}_{5}$ platelets (flakes) approximately $100 \mathrm{~nm}$ thick and with an aspect ratio as high as $10^{2}-10^{3}$ were obtained. The nanoflakes were susceptible to re-crystallization annealing and interestingly exhibited a room-temperature coercivity $(H c)$ up to $H c=$ $19 \mathrm{kOe}[3]$.

\footnotetext{
* Corresponding author. Tel: (34) 913366569

Email address: d.rojas@upm.es (D. P. Rojas).
}

The high-energy ball milling production route has also been successfully applied in the study of other Re-based alloys. In this regard, magnetization measurements on a series of samples of cubic $\mathrm{TbFe}_{2}$ alloys with different milling times have shown an abrupt increase of the coercivity from $H c=0.2 \mathrm{kOe}$ up to $H c=6.7 \mathrm{kOe}$ at $300 \mathrm{~K}$ with the increase of the milling time up to $51 \mathrm{~h}$ [4]. It is worth noticing that most of the reports, including those above, have been focused on the coercivity behavior, whereas much lower number of studies have been devoted to the analysis of the dynamics of AC-susceptibility in Re systems. This is awkward as it is obvious that the milling procedure usually induces the presence of magnetic disorder and eventually the production of cuasi-spherical magnetic particles. In this sense, it is phenomenal that even bulk polycrystalline $\mathrm{GdAl}_{2}, \mathrm{DyAl}_{2}$, and $\mathrm{ErAl}_{2}$ are affected by particular domain configurations giving rise in measurements of the AC magnetic susceptibility to a especial spin dynamics [5]. In $\mathrm{Sm}_{2} \mathrm{Fe}_{7}$ alloy, 
an anomalous rise in the real component $\left(\chi^{\prime}\right)$ is explained by dynamic domain-wall pinning and depinning, and as a consequence of thermally activated local directional ordering of Fe atoms [6]. Systematic magnetic AC-susceptibility ( $\chi a c$ ) measurements on several $\mathrm{Re}_{2} \mathrm{Fe}_{14} \mathrm{BH}_{x}$ compounds have revealed the existence of mobile defects coupled to the domain walls [7]. From these works, it turns out that the analysis of AC-susceptibility dynamics is important to accomplish a more complete characterization of the magnetic behavior of these systems.

However, it is surprising that to date, even these few studies were constrained to bulk samples, and a little attention has been paid to nanosized Re-based alloys. The first step following this direction was undergone by studying nanocrystalline $\mathrm{TbAl}_{2}$ alloys obtained by mechanical milling $[8,9]$. In this system, AC- and DC-magnetic susceptibility results between 5 and $300 \mathrm{~K}$ showed that the longrange ferromagnetic $(\mathrm{FM})$ structure $\left(T_{C}=105 \mathrm{~K}\right)$ in the bulk sample is inhibited in favor of a disordered spin arrangement below $T=45 \mathrm{~K}$ in the milled samples [8]. In addition, several frequency dependent contributions, below the freezing temperature, both in real and imaginary components of $\chi a c$ (especially in $300 \mathrm{~h} \mathrm{TbAl}_{2}$ milled alloy) were also observed. The possibility of tuning the magnetic disorder by milling is then attractive and could be combined with other research routes. A feasible possibility to deepen in the properties of an Re-alloy is to dilute such a parent alloy with another element. This idea has been followed recently with the study of X-ray magnetic circular dichroism at the K-edge of $\mathrm{Tb}\left(\mathrm{Al}_{1-x} \mathrm{Fe}_{x}\right)_{2}$ intermetallics. These experiments have shown that aluminium substitution affects only slightly the magnitude of the individual $\mathrm{Tb}$ and $\mathrm{Fe}$ magnetic moments but strongly reduces the exchange interactions [10].

Considering the above results, the present aim of this work is to study of the influence of the mechanical milling on the magnetic properties of bulk $\mathrm{TbAl}_{1.5} \mathrm{Fe}_{0.5}$ alloy. The expected reduction of particle size will surely influence the exchange coupling and anisotropy in the alloy. Our analysis will be focused on the frequency dependence of ACmagnetic susceptibility and the coercivity variations. The effect of the Fe-dilution/milling process will be discussed and compared with earlier results reported in neighbor $\mathrm{TbAl}_{2}$ nanometric alloys [8] and very similar Re-alloys [5].

\section{Experimental details}

The starting polycrystalline $\mathrm{TbAl}_{1.5} \mathrm{Fe}_{0.5}$ pellet was prepared by arc melting suitable amounts of pure constituents $\mathrm{Tb}$ (3N-Alfa), Al (5N-Alfa) and Fe (3N-Alfa), in an arc furnace under protective Ar atmosphere. A mass of $5 \mathrm{~g}$ of this alloy was milled during 25 and 40 hours (allowing 1 hour cool down after each one hour milling time) inside a planetary high energy ball system Retsch PM 400/2 at a rotation speed of $200 \mathrm{rpm}$, using an air-tight container and balls made of tungsten carbide, also under Ar gas. The struc- tural characterization was performed by X-ray diffraction in a Philips PW1710 diffractometer, using $\mathrm{Cu}-\mathrm{K}_{\alpha}$ radiation. The magnetic properties, DC-magnetization $M(H, T)$ and AC-susceptibility were collected with a Quantum Design PPMS multipurpose instrument in the 2-300 K temperature range and occasionally applying magnetic fields $(H)$ up to $H=90 \mathrm{kOe}$. Mössbauer spectrum was measured at room temperature by using a constant acceleration spectrometer which uses a rhodium matrix source and was calibrated with an $\alpha$-Fe foil. The Mössbauer spectral absorbers contained approximately $35 \mathrm{mg} / \mathrm{cm}^{2}$ of powdered sample which had been sieved to a $0.045 \mathrm{~mm}$ or smaller diameter of particle size.

\section{Results}

$\mathrm{X}$-ray diffraction patterns in bulk and milled $\mathrm{TbAl}_{1.5} \mathrm{Fe}_{0.5}$ alloys are presented in Figure 1. A broadening and a reduction of the maximum intensity of the diffraction peaks, associated to the decrease of the size of the starting polycrystalline particles and the increase of the lattice strain, are observed. The diffraction patterns are consistent with a cubic $F d \overline{3} m$-type structure (see inset of Figure 1), and no extra phases were detected. The lattice parameter $(a)$ does not change significantly with the milling process (see Table 1). Using the Williamson-Hall procedure,[11] values for mean size $(D)$ of the particles and lattice strain $(\eta)$ were estimated, and the results are also presented in Table 1. In the table it can also be deduced a marginal increase of the lattice parameter $(0.0025 \AA)$. These parameters follow the expected tendency of a decrease of size and a concomitant increase of the lattice strain with the increase of the milling time, as was discussed in detail in the series of $\mathrm{TbAl}_{2}$ milled alloys [8] and many other nanostructured metallic alloys produced via high-energy milling [12]. It is then expected that the change of structure produced by milling with reduction of particle size and the increase of disorder deduced from the high value of strain will originate changes in the anisotropy and spin dynamics. In this sense, it is remarkable that a (relative) short period time $(25 \mathrm{~h})$ is enough to produce such small particles, well-below the single domain limit for typical Re [8], probing the usefulness of the milling route to achieve sizes within such a limit.

Table 1

Values of lattice parameters $(a)$, mean size $(D)$ and strain $(\eta)$ for bulk and milled $\mathrm{TbAl}_{1.5} \mathrm{Fe}_{0.5}$ alloys.

\begin{tabular}{lccc}
\hline Sample & $a(\AA)$ & $D(\mathrm{~nm})$ & $\eta(\%)$ \\
\hline Bulk & $7.7590(1)$ & - & - \\
$25 \mathrm{~h}$ & $7.7615(2)$ & $13(1)$ & $1.1(0.2)$ \\
$40 \mathrm{~h}$ & $7.7613(2)$ & $10(2)$ & $1.7(0.3)$ \\
\hline
\end{tabular}




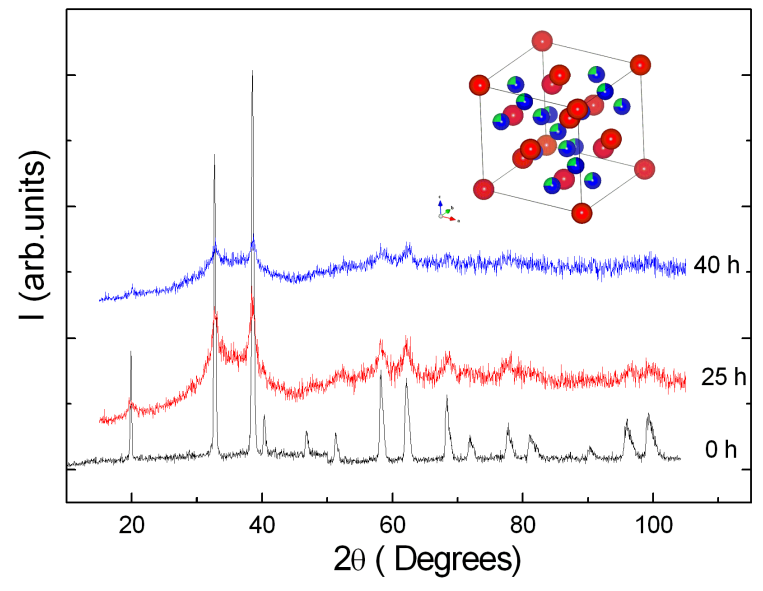

Fig. 1. X-ray diffraction patterns of bulk and milled $\mathrm{TbAl}_{1.5} \mathrm{Fe}_{0.5}$ alloys for two milling times (spectra have been shifted up for clarity). A progressive broadening and reduction of the intensity of the peaks with the increase of the milling time are observed. In the inset, the crystallographic structure corresponding to the cubic $F d \overline{3} m$-type is depicted.

\subsection{Magnetic properties of bulk $\mathrm{TbAl}_{1.5} \mathrm{Fe}_{0.5}$ alloy}

In order to address the effects of Fe-dilution on the magnetic properties of $\mathrm{TbAl}_{2}$ bulk alloy, which is $\mathrm{FM}$ at 105 $\mathrm{K}$ [8], measurements of the thermal variation of the DCmagnetic susceptibility at $H=100$ Oe, are presented in Figure 2, for bulk $\mathrm{TbAl}_{1.5} \mathrm{Fe}_{0.5}$ alloy (henceforth, referred as 0 $\mathrm{h}$ ). For these measurements, the alloy was first cooled from $300 \mathrm{~K}$ to $2 \mathrm{~K}$ under no applied magnetic field (zero-field cooled regime, ZFC), and then with a $H=100$ Oe (fieldcooled regime, FC). Afterwards, the susceptibility curves were collected on warming. The FC variation increases with decreasing temperature as in an ordinary ferromagnet. The inset details the temperature dependence of the first derivative, showing a minimum at $T=114 \mathrm{~K}$, associated to a FM ordering. This behavior is similar to that found in the parent $\mathrm{FM}$ alloy $\mathrm{TbAl}_{2}$, with $T_{C}=105 \mathrm{~K}$. This increase of the $\mathrm{FM}$ transition caused by the $\mathrm{Al}$ replacement by Fe atoms is expected, as precisely in $\mathrm{TbFe}_{2}$ alloy, the value of $T_{C}=703$ $\mathrm{K}$ [4]. The FM ordering in $0 \mathrm{~h}$ alloy is further supported by measurements of the magnetic field dependence of magnetization at $(T=5 \mathrm{~K})$, showing a characteristic FM shape with a linear behavior in the high-field region (see Figure 3). The linear increase of magnetization (high-field) may be indicating may be indicative of crystal electric field effects associated to $\mathrm{Tb}$-ions or non-collinearity. A precise confirmation would require of further microscopic evidence, namely neutron diffraction and Mösbauer spectroscopy. In any case, the ferromagnetic signal is further confirmed by the Arrott plots $\left(M^{2}\right.$ vs. $\left.H_{\text {int }} / M\right)$ at temperatures between 100 and $200 \mathrm{~K}$. After the correction from the demagnetizing effects, the plots yield straight isotherms with an intercept tending to zero when approaching the Curie temperature. A typical FM state is observed, obeying the Weiss molecularfield theory [13], as deduced from inspection of Figure 4. An extrapolation of the high-field slopes gives an estimate for the $T_{C}=115.0(2.5) \mathrm{K}$, in fair agreement with the DCmagnetic susceptibility results. This FM phase is however, affected by a high (at $T=5 \mathrm{~K},(M / H)_{F C}-(M / H)_{Z F C}=$ $10 \mathrm{emu} / \mathrm{molOe}$, around $90 \%$ of the $M / H$ value) irreversibility, which is observed between the ZFC and FC curves. In addition, the ZFC susceptibility shows an extra maximum around $T=60 \mathrm{~K}$, followed by a decrease of its value when approaching the lowest temperature. This fact is very similar to the $\mathrm{TbAl}_{2}$ case and the irreversibility should be caused by the existence of small domains (clusters) which become progressively magnetically frustrated during the cooling process.

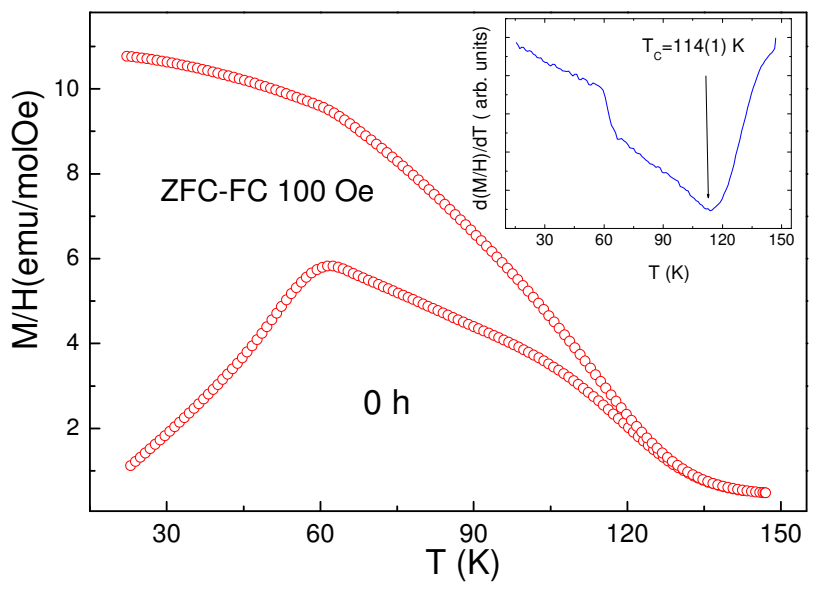

Fig. 2. Temperature dependence of the ZFC and FC curves of DC magnetic susceptibility at $H=100 \mathrm{Oe}$ in the bulk $\mathrm{TbAl}_{1.5} \mathrm{Fe}_{0.5}$ sample. The inset details the first derivative indicating the minimum, and consequently, the value of $T_{C}=114 \mathrm{~K}$.

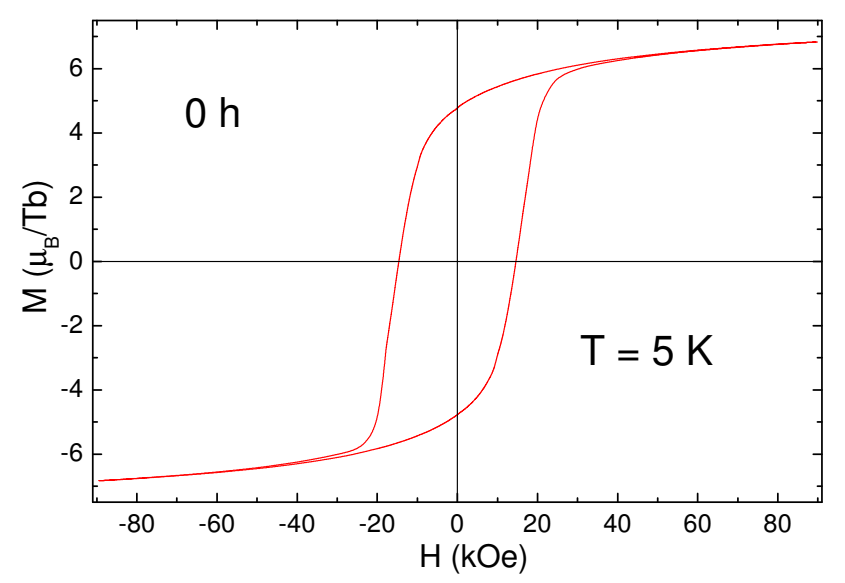

Fig. 3. Hysteresis loop of the bulk $\mathrm{TbAl}_{1.5} \mathrm{Fe}_{0.5}$ alloy $(0 \mathrm{~h}$ milled alloy) at $T=5 \mathrm{~K}$.

The study of the spin dynamics has been carried out using $\chi a c$ measurements at different frequencies, ranging from $10 \mathrm{~Hz}$ to $10 \mathrm{kHz}$, and in the temperature range between $5 \mathrm{~K}-125 \mathrm{~K}$, as shown in Figure 5. It is observed that the FM transition shifts up to higher temperatures from $105 \mathrm{~K}$ in $\mathrm{TbAl}_{2}$ to $114 \mathrm{~K}$ in $\mathrm{TbAl}_{1.5} \mathrm{Fe}_{0.5}$, confirming the DC-magnetization data. This fact points towards a definite strengthening of the exchange interactions with the 


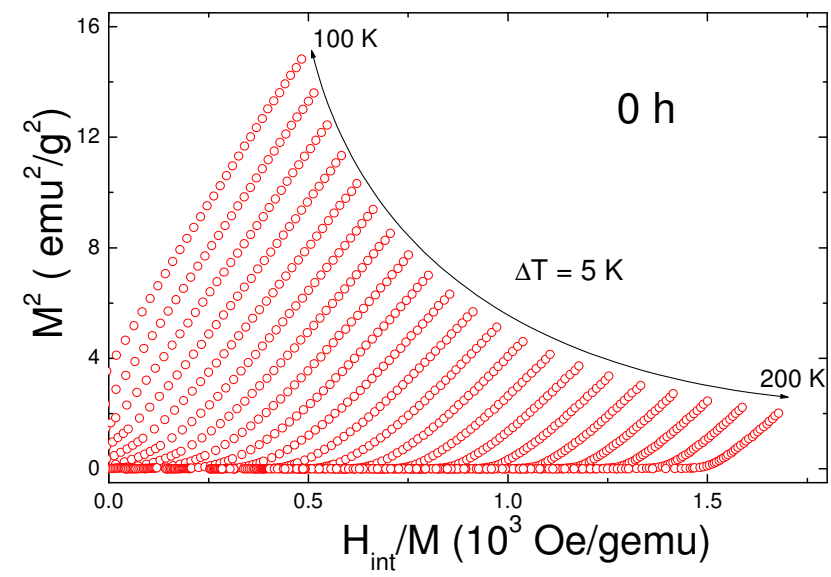

Fig. 4. Arrott plots $M^{2}$ vs $H_{\text {int }} / M$ of the bulk $\mathrm{TbAl}_{1.5} \mathrm{Fe}_{0.5}$ alloy. The intercept of the high field region gives an estimate of the $T_{C}=115.0(2.5) \mathrm{K}$.

Fe-substitution, as reported previously [10]. In addition, a broad peak appears around $70 \mathrm{~K}$. This additional contribution below $T_{C}$ was also observed in bulk $\mathrm{TbAl}_{2}$ alloy [8]. In such a binary alloy, the frequency dependence was consistent with a domain wall motion, described by an Arrhenius thermal activation process. This description coincides with that reported for FM bulk alloys of $\mathrm{DyAl}_{2}$ and $\mathrm{Sm}_{2} \mathrm{Fe}_{7}[5,6]$.

In the present Fe-containing alloy, the frequency dependence of the susceptibility peak can be described uniquely by the well-known Vogel-Fulcher (VF) law. The latter differs from the Arrhenius activation law as it incorporates an interaction temperature $T_{0}$. The values obtained from the fitting give $T_{0}=66 \mathrm{~K}$ and activation energy $E_{a}=276 \mathrm{~K}$. The VF phenomenological law is usually applied to glassy dynamics [14]. Furthermore, the value for the relative shift per frequency decade using $T_{0}$ as the peak temperature, $\delta=\Delta T_{f} / T_{f} \log \nu=0.0143$, is between those reported for canonical spin glasses (SG) and superparamagnetic (SPM) systems $[14,15]$.

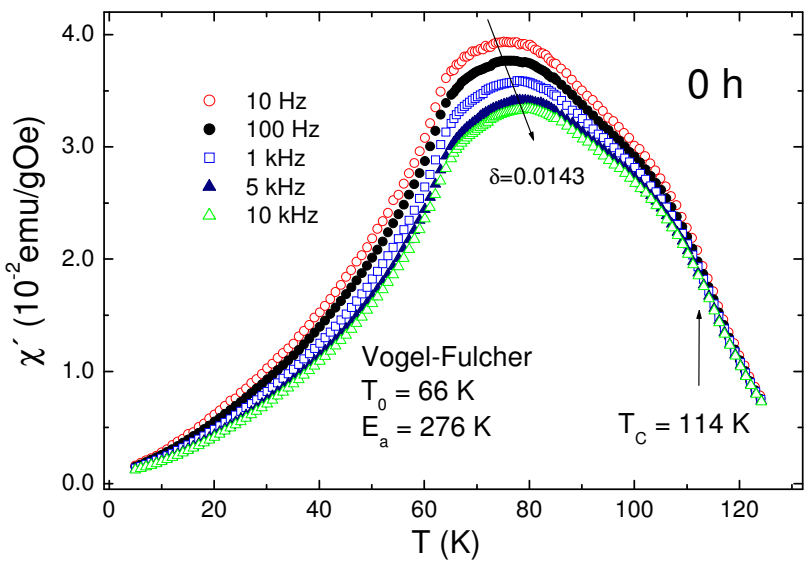

Fig. 5. Temperature dependence of the real component $\left(\chi^{\prime}\right)$ of $\mathrm{AC}-$ magnetic susceptibility at different frequencies for bulk $\mathrm{TbAl}_{1.5} \mathrm{Fe}_{0.5}$ alloy. The ferromagnetic transition (at $114 \mathrm{~K}$ ) is higher than in $\mathrm{TbAl}_{2}$ binary alloy $\left(T_{C}=105 \mathrm{~K}\right)$. The peak around $65 \mathrm{~K}$ shows glassy behavior obeying the Vogel-Fulcher law.
In Re intermetallics containing transition metals as Fe, it is essential to elucidate the eventual ability of Fe-atoms to carry a magnetic moment, and hence their contribution to the overall magnetic behavior of the given system. In our $\mathrm{TbAl}_{1.5} \mathrm{Fe}_{0.5}$ alloy with a low Fe-concentration, it is likely that there is a modification of the $\mathrm{Fe}$ and $\mathrm{Al}$ bands, resulting in a non-magnetic character of the Fe. In fact, the data stemming from the magnetic and Mössbauer measurements on the series of $\mathrm{Y}\left(\mathrm{Fe}_{x} \mathrm{Al}_{1-x}\right)_{2}$ alloys, with $0<$ $x \leq 0.8$ showed that the long-range magnetic order associated to the Fe, disappears at about $x=0.78$. Additionally, Mössbauer spectroscopy results on $\mathrm{Y}\left(\mathrm{Fe}_{0.22} \mathrm{Al}_{0.78}\right)_{2}$ (for $x=0.22$ ), with a Fe-concentration very near to that of our Tb-based alloy $(x=0.25)$ showed no magnetic hyperfine splitting in zero field down to $\mathrm{T}=4.2 \mathrm{~K}$ [16]. Moreover, the non-magnetic state of Fe in Re systems is not an uncommon phenomenon; it has also been observed, for instance, in the series of $\mathrm{DyFe}_{x} \mathrm{Sn}_{2}$ alloys [17].

In order to check the magnetic state of $\mathrm{Fe}$ in $0 \mathrm{~h}$ alloy (or $\mathrm{Tb}\left(\mathrm{Al}_{0.75} \mathrm{Fe}_{0.25}\right)_{2}, x=0.25$ ), measurements of the Mössbauer spectrum at $295 \mathrm{~K}$ were carried out, and the results are displayed in Figure 6. At this temperature the Mössbauer spectra consists of a broad doublet, reflecting that the compound is in a paramagnetic state, as observed in the Y-series of alloys [16]. It is known that in the $\operatorname{Re}\left(\mathrm{Fe}_{x} \mathrm{Al}_{1-x}\right)_{2}$ compounds, the aluminum atoms are randomly distributed over the $16 d \mathrm{Fe}$ sites. As a consequence, seven different nearest neighbor environments are possible for an iron site, and each iron atom surrounded by a given environment will contribute to the total Mössbauer spectrum with a relative intensity given by the probability associated to such an environment. According to this, seven different doublets should be used to fit the paramagnetic spectrum. Clearly, this strict analysis results in an excess of adjustable parameters and the physical reliability is missing. For this reason, only one doublet was used to fit the Mössbauer spectrum of $0 \mathrm{~h}$ alloy yielding the values of $I S=0.098 \pm 0.02 \mathrm{~mm} / \mathrm{s}$, for the isomer shift, and $\Delta E_{Q}=$ $0.226 \pm 0.02 \mathrm{~mm} / \mathrm{s}$, for the quadrupole splitting. In particular, the value obtained for the isomer shift is near to that reported in $\mathrm{Y}\left(\mathrm{Fe}_{0.22} \mathrm{Al}_{0.78}\right)_{2}$ alloy of $0.13 \mathrm{~mm} / \mathrm{s}[16]$.

\subsection{Magnetic properties of milled $\mathrm{TbAl}_{1.5} \mathrm{Fe}_{0.5}$ alloys}

The influence of the milling process on the magnetic properties of $0 \mathrm{~h}$ alloy has been studied in two samples, obtained after $25 \mathrm{~h}$ and $40 \mathrm{~h}$ of milling time, and with a mean grain size of $D=13 \mathrm{~nm}$ and $D=10 \mathrm{~nm}$, respectively (see Table 1). There are similar common characteristic for both samples regarding the static DC-magnetization and AC-susceptibility. We will start the description with the 25 $\mathrm{h}$ milled alloy showing in Figure 7 the static results. At low magnetic fields ( $H=100 \mathrm{Oe}$ ) (Figure $7 \mathrm{a})$, the ZFC and the FC curves present a large irreversibility, similar to that observed in bulk alloy. In particular, the ZFC curve shows two contributions at $T_{\max }=63 \mathrm{~K}$ (commonly referred in 


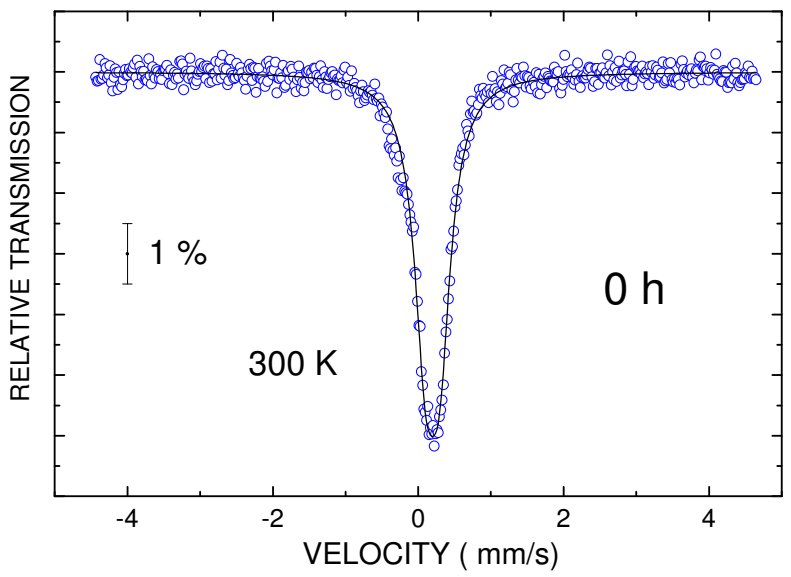

Fig. 6. Mössbauer spectrum of the bulk $\mathrm{TbAl}_{1.5} \mathrm{Fe}_{0.5}$ alloy taken at $T=300 \mathrm{~K}$. The solid line is a fit according to the contribution of a paramagnetic doublet.

the literature as the irreversibility temperature) and $T^{*}=$ $33 \mathrm{~K}$. Increasing the magnetic field, the peak at $T_{\max }$ shifts down to lower temperatures and merges with the contribution corresponding to $T^{*}$ into a single peak, at $H=3.5$ $\mathrm{kOe}$ (see Figure $7 \mathrm{~b}$ ). The inset of Figure $7 \mathrm{~b}$ shows the field dependence of the high temperature peak $\left(T_{\max }\right)$, according to a $H^{2 / 3}$ dependence, commonly encountered in SG systems [14].

Following the description of results, we now focus on the temperature dependence of $\chi^{\prime}$ of $25 \mathrm{~h} \mathrm{TbAl}_{1.5} \mathrm{Fe}_{0.5}$, presented in Figure 8. The shape of this curve is similar to the ZFC one at $H=100$ Oe, with two maxima around $65 \mathrm{~K}$ and $30 \mathrm{~K}$, pointing to the presence of two different magnetic states. The peak at around $65 \mathrm{~K}$ presents different parameters to the bulk state, namely, the interaction temperature $T_{0}=63 \mathrm{~K}$ and the activation energy $E_{a}=109 \mathrm{~K}$, according to the fitting to the above mentioned VF law. The value of the relative shift per frequency decade, $\delta=0.0055$, is now closer (respect to the bulk value, see Table 2) to that reported for spin glasses [15]. Additionally, the contribution appearing around $T^{*}=33 \mathrm{~K}$, also shifts up to higher temperature with the increase of the frequency. Details of this frequency dependent peak are shown in the inset, for the imaginary component $\left(\chi^{\prime \prime}\right)$. The relative shift $\delta^{*}=0.1$ (see inset of Figure 8) is much larger than commonly observed in canonical spin glasses, but it is similar to that reported in insulating SG systems like $(\mathrm{Fe}, \mathrm{Mg}) \mathrm{Cl}_{2}(\delta=0.08)$, and lower than classical SPM systems $\left(\mathrm{Ho}_{2} \mathrm{O}_{3}-\mathrm{B}_{2} \mathrm{O}_{3}, \delta=0.3\right)$ [14].

Turning our attention to the $40 \mathrm{~h}$ milled alloy, we again first begin with the analysis of the static ZFC curves of DCmagnetic susceptibility in Figure 9. These also evidence the presence of two maxima as in the former case and $T_{\max }$ also obeys the $H^{2 / 3}$ field dependence, although somewhat affected by the low temperature contribution at $T=30 \mathrm{~K}$, as indicated in the inset of Figure 9 . The $M(H)$ dependence (Hysteresis loop) is shown in Figure 10 for the $40 \mathrm{~h}$ milled alloy at $T=5 \mathrm{~K}$. The most relevant result is the reduction

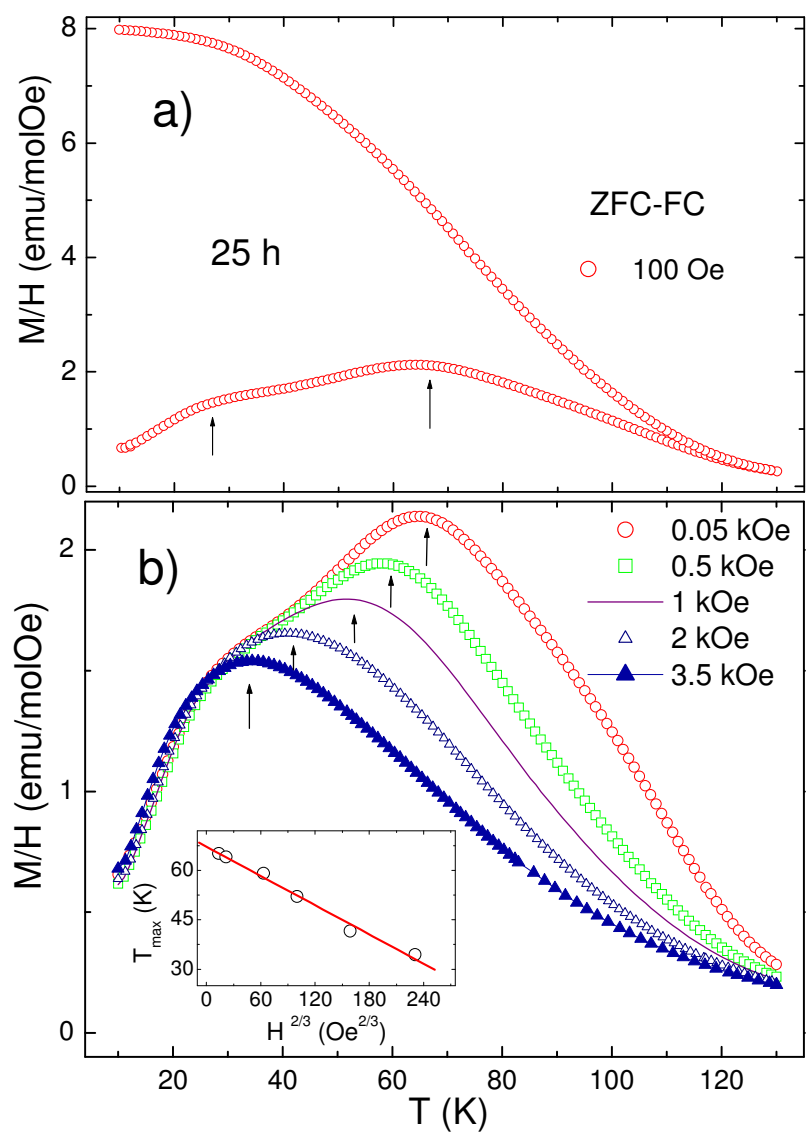

Fig. 7. a)Temperature dependence of ZFC and FC curves of DCmagnetic susceptibility at $H=0.1 \mathrm{kOe}$ for the $25 \mathrm{~h}$ milled alloy. The arrows show the contributions at $T_{\max }$ and $T^{*}$ in agreement with AC-susceptibility results. b) ZFC curves vs temperature at different magnetic fields. The high temperature maximum follows the $H^{2 / 3}$ variation characteristic of $\mathrm{SG}$, as depicted in the inset.

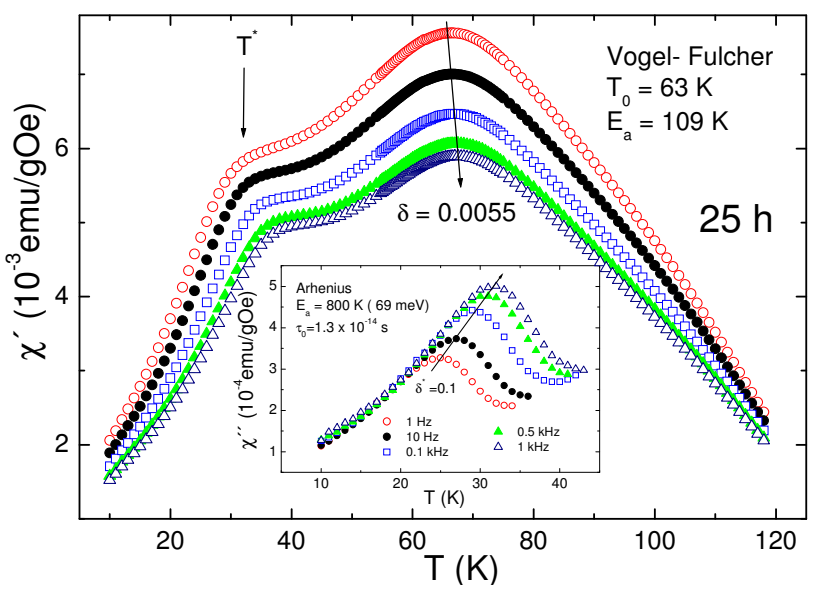

Fig. 8. $\chi^{\prime}$ vs temperature for $25 \mathrm{~h}$ milled alloy. Both a peak with a glassy behavior at $T=63 \mathrm{~K}$ and additional anomaly around $T^{*}$ $=33 \mathrm{~K}$ are observed. The inset details the frequency dependence of the imaginary component around $T^{*}$, which can be described by an Arrhenius-type activation process.

of $H c=15 \mathrm{kOe}$ in the bulk sample down to $1 \mathrm{kOe}$ in the milled alloy. This reflects that the Tb-Al-Fe milled alloys consist of nanometric grains with sizes inside the single- 
domain region, similar to that observed in $\mathrm{TbAl}_{2}$ milled alloys [8]. The Arrott plots represented in Figure 11 show a change of curvature between the low temperature region 10 $\mathrm{K} \leqslant \mathrm{T} \leqslant 54 \mathrm{~K}$, and the high temperature $52 \mathrm{~K} \leqslant \mathrm{~T} \leqslant 108 \mathrm{~K}$. This change should related to the mentioned existence of a disordered arrangement of single domain nanoparticles.

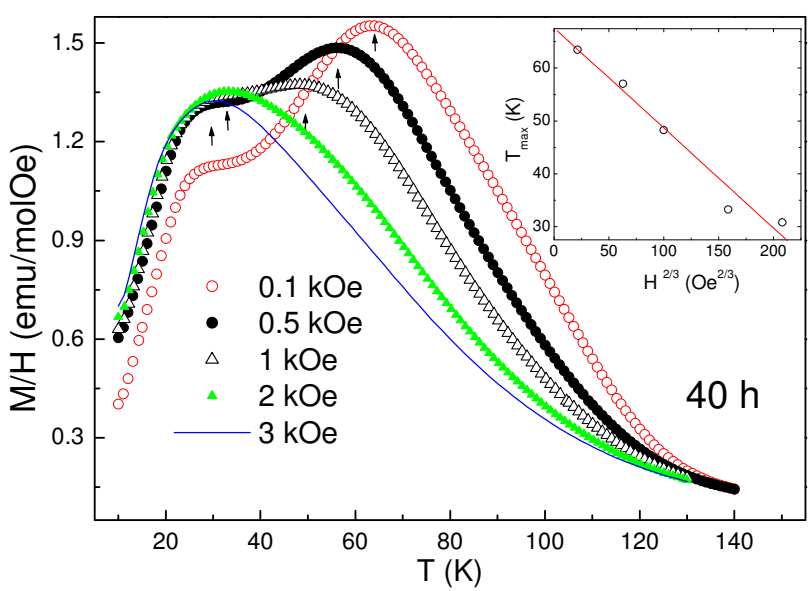

Fig. 9. Temperature dependence of ZFC curves of DC-magnetic susceptibility at different magnetic fields for $40 \mathrm{~h}$ milled alloy. The high temperature maximum follows a $H^{2 / 3}$ variation characteristic of SG, although it is somewhat affected by the presence of the second maximum at lower temperatures.

The field dependence of the magnetization for $25 \mathrm{~h}$ and 40 $\mathrm{h}$ - milled $\mathrm{TbAl}_{1.5} \mathrm{Fe}_{0.5}$ alloys can be compared with that of the bulk alloy, as shown in Figure 12. A decrease of the magnetization, for instance, at $H=90 \mathrm{kOe}$, of the milled samples respect to the bulk one, is observed (see the values of saturation magnetization $\left(M_{S}\right)$ in Table 2). Although, this reduction is more abrupt from bulk to the $25 \mathrm{~h}$ milled alloy, a general tendency is conserved. This behavior is similar to that observed in $\mathrm{TbAl}_{2}$ milled alloys. Moreover, the highfield magnetization slope is increased respect to the bulk alloy. This is a sign of a definite spin canting in the nanometric alloys due to the specific large surface/volume ratio increasing the surface spins with a random anisotropy. Consequently, it costs more energy to overcome the anisotropy energy. Another important result is that the analysis of the hysteresis curves (see Figures 3 and 10) allows to estimate the values of $H c$, and these have been inserted in Table 2 . The coercivity of bulk $\mathrm{TbAl}_{1.5} \mathrm{Fe}_{0.5}$ increases 200 times, approximately, when Fe partially substitutes $\mathrm{Al}$ in the $\mathrm{TbAl}_{2}$ alloy.

Respect the spin dynamics in the $40 \mathrm{~h}$ alloy, it is possible to state that: i) the presence of the two transitions observed in the DC-susceptibility is further confirmed in the $\chi$ ac curves presented in Figure 13, and ii) the frequency variation produces a shift to higher temperatures in both peaks, with very different relative shift values. In the inset, the variation of the low temperature peak is expanded.

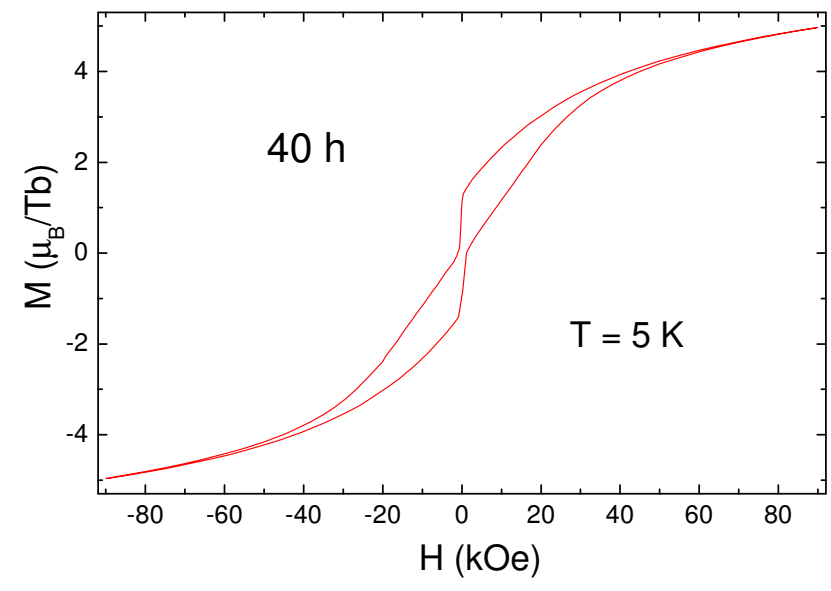

Fig. 10. Hysteresis loop of the $40 \mathrm{~h}-$ milled alloy at $5 \mathrm{~K}$.
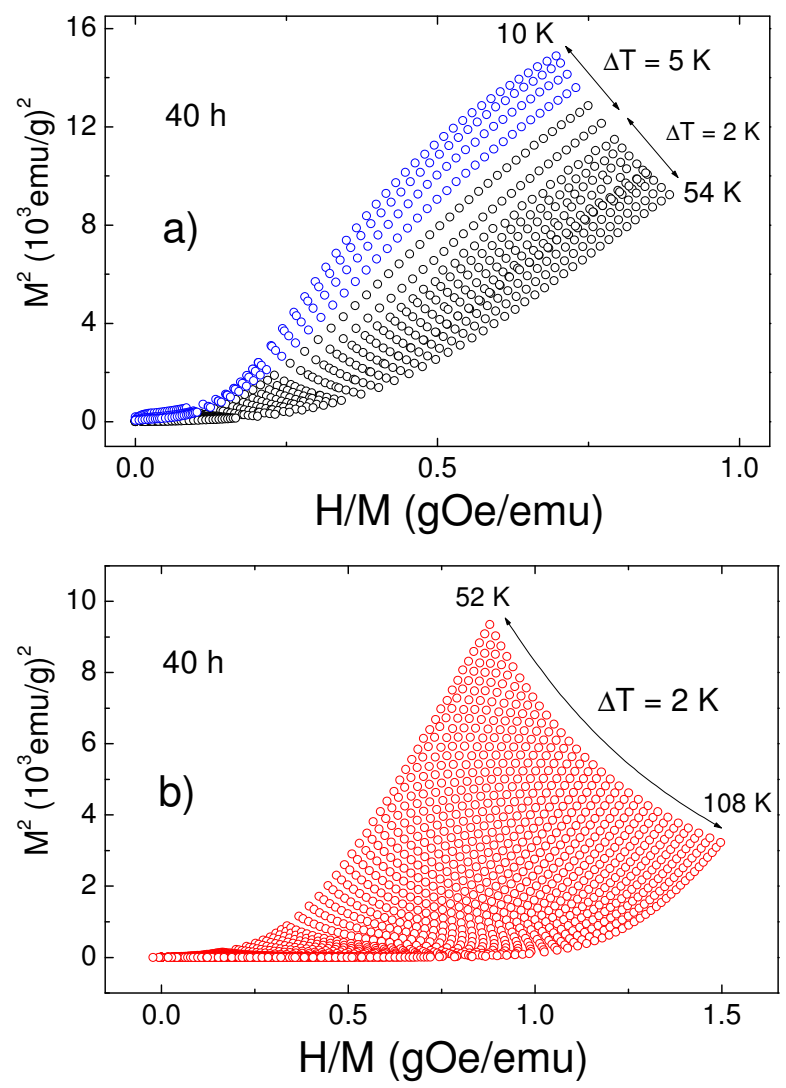

Fig. 11. Arrott plot $\mathrm{M}^{2}$ vs $\mathrm{H}_{\text {int }} / \mathrm{M}$ of the $40 \mathrm{~h}$ milled alloy. a) Low temperature region $10 \mathrm{~K} \leqslant \mathrm{~T} \leqslant 54 \mathrm{~K}$, and $\mathrm{b}$ ) high temperature 52 $\mathrm{K} \leqslant \mathrm{T} \leqslant 108 \mathrm{~K}$. A change in the curvature on going for one region to another is observed.

\section{Discussion}

The described results of the static and dynamic magnetic properties of $\mathrm{TbAl}_{1.5} \mathrm{Fe}_{0.5}$ alloys in bulk and nanocrystalline state allows to reveal in detail the particle size influence. The bulk alloy additionally acts as a control sample helping to understand the size modifications. Briefly, decreasing the temperature from $300 \mathrm{~K}$, the bulk (parent) 
Table 2

Values of the saturation magnetization $\left(M_{S}\right)$, coercivity $\left(H_{C}\right)$, ferromagnetic transition $\left(T_{C}\right)$, glassy transition $\left(T_{0}\right)$, and relative shift for bulk and milled $\mathrm{TbAl}_{1.5} \mathrm{Fe}_{0.5}$ alloys.

\begin{tabular}{lcccccc}
\hline Sample & $M_{\text {sat }}\left(\mu_{B} / \mathrm{Tb}\right)$ & $H_{C}(\mathrm{kOe})$ & $T_{C}(\mathrm{~K})$ & $T_{g}(\mathrm{~K})$ & $\delta$ & $\delta^{*}$ \\
\hline Bulk & $7.39(1)$ & $14.60(5)$ & $112(1)$ & $66(1)$ & 0.0143 & - \\
$25 \mathrm{~h}$ & $6.27(2)$ & $7.14(5)$ & - & $63(1)$ & 0.0055 & 0.1 \\
$40 \mathrm{~h}$ & $6.04(2)$ & $1.04(2)$ & - & $61(1)$ & 0.0056 & 0.1 \\
\hline
\end{tabular}

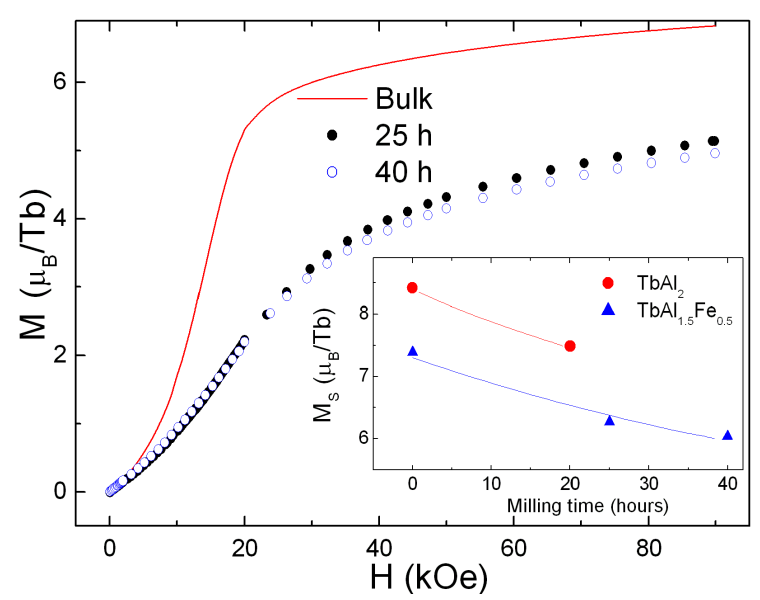

Fig. 12. Field dependence of the magnetization for bulk, $25 \mathrm{~h}$, and 40 $\mathrm{h}$ milled alloys. Inset: Similarly to $\mathrm{TbAl}_{2}$ milled alloys, a reduction of the saturation magnetization $\left(M_{S}\right)$ with the increase of the milling time is found.

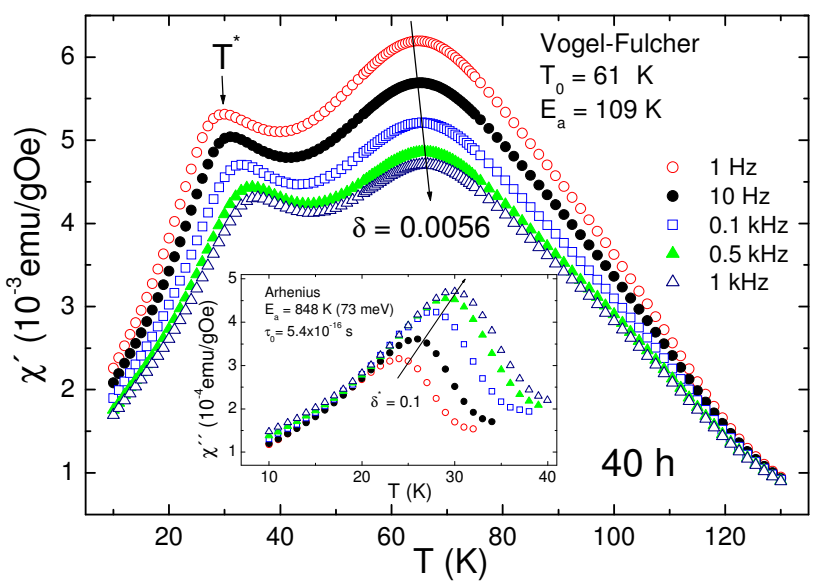

Fig. 13. $\chi^{\prime}$ vs temperature for $40 \mathrm{~h}$ milled alloy. Both a peak with a glassy behavior at $61 \mathrm{~K}$ and additional anomaly around $T^{*}=30$ $\mathrm{K}$ (more pronounced than in $25 \mathrm{~h}$ milled alloy) are observed. The inset details the frequency dependence of the imaginary component around $T^{*}$.

alloy presents a magnetic behavior starting from a paramagnetic phase, microscopically evidenced by Mössbauer. Around $T=114 \mathrm{~K}$, the alloy enters into a FM state, which is present still at $\mathrm{T}=5 \mathrm{~K}$, as understood from the $M(H)$ behavior. The magnetic contribution should stem from $\mathrm{Tb}$ and it is expected that for such low Fe-concetrations the Featoms remain in a non-magnetic due to changes in the electronic band structure of $\mathrm{Al}$ and Fe. [16]. However, around $60 \mathrm{~K}$, both the DC- and AC-measurements mark the presence of a new (clustered) phase with the presence of a glassy dynamics, which is still mixed with a remaining FM contribution. This behavior resembles that reported on $\mathrm{TbAl}_{2}$ alloy, the latter showing a couple of contributions at 105 $\mathrm{K}$ and around $44 \mathrm{~K}$ as well. There are though two distinctions: firstly, in the present Fe-substituted alloy, the $T_{C}$ is slightly higher. This is expected due to the enhancement of exchange interactions thanks to Al substitution by Featoms, and with an increasing tendency to a crossover to a high $T_{C}$ value of $703 \mathrm{~K}$, reported in $\mathrm{TbFe}_{2}$ alloy [4]. Secondly, the magnitude of the contribution at $T_{C}$ is reduced and the lower temperature peak shows glassy features rather than strict domain wall dynamics observed $\mathrm{TbAl}_{2}$ [8], following the AC-susceptibility results. As a consequence, the random substitution of $\mathrm{Al}$ by $\mathrm{Fe}$ increases the random anisotropy and promotes a low-temperature glassy behavior. In fact, it looks as if the $\mathrm{TbAl}_{1.5} \mathrm{Fe}_{0.5}$ alloy is closer to $300 \mathrm{~h}$ milled $\mathrm{TbAl}_{2}$, with lower characteristic VF parameters of $T_{0}(41 \mathrm{~K}), E_{a}(64 \mathrm{~K})$, and $\delta=0.0026$, closer to that observed in canonical SG systems [8]. Overall, the bulk alloy behaves according to a reentrant ferromagnet, in which there is a transition from a FM to a spin glass state, influence by ferromagnetically coupled regions acting as small domain (clusters) $[14,15,18]$. In this sense, it is then critical to define the spin correlation length of the domains; the delicate balance between exchange and anisotropy may reduce their size, resulting in an heterogeneous spin arrangement [19].

Regarding the $25 \mathrm{~h}$ and $40 \mathrm{~h}$ milled alloys, the FM contribution tends to disappear gradually and glassy peaks are observed at $T_{0}=63 \mathrm{~K}$ and $61 \mathrm{~K}$, respectively. Respect to the bulk sample, the VF activation energy decreases from $E_{a}=276 \mathrm{~K}$ to $109 \mathrm{~K}$ and the value of the relative shift per frequency decade $\delta$, from 0.0143 to 0.0055 . The latter values are indicating that the milling process favors the disorder, and consequently a magnetic glassy behavior close to those in archetypal SG systems. This process is favored by the presence of magnetic nanoparticles, which constitute the structural basis for the disordered structure. In fact, a remaining FM behavior is explained due to the proximity of the particles, enabling a effective exchange connection among them [20]. In short, there is a disordered state giving rise to the glassy $T_{0}$ peaks with some scattered FM coupling among the particles.

The low temperature contribution, labeled as $T^{*}$, around $30 \mathrm{~K}$ in both milled samples does not stem from a possible Fe segregation, as observed in other Fe-containing ( $\mathrm{Tb}-\mathrm{Fe}-$ B) milled alloys, because in our Tb-Al-Fe alloys the magnetization does not increase when milling the samples [21]. Interestingly, these low temperature contributions, below the glassy peak, have also been observed in $300 \mathrm{~h} \mathrm{TbAl}_{2}$ milled alloy, with a relative shift $\delta=0.09$, similar to that observed here for the Tb-Al-Fe milled alloys. On the one hand, the frequency dependence of the peak of these contributions cannot be described by a Vogel-Fulcher law, with physical meaning parameters. On the other hand, the value of the relative shift $\left(\delta^{*}=0.1\right)$ has been found in systems of single domain magnetic nanoparticles, ascribed in the 
SPM regime [15]. In the same way, similar values have been reported in various other magnetic systems, with coexistence of different magnetic phases, namely, antiferromagnetic and SG states [22], SPM-SG-FM behaviors [23], and cluster glass CG-SPM [24]. In this sense, the analysis of the frequency dependence of the low temperature contributions in $25 \mathrm{~h}$ and $40 \mathrm{~h} \mathrm{TbAl}{ }_{1.5} \mathrm{Fe}_{0.5}$ milled alloys formally fits to an Arrhenius law. However, the calculated parameters yield unphysical relaxation times $\tau_{0}=1.3 \cdot 10^{-14} \mathrm{~s}$ and $\tau_{0}=5.4 \cdot 10^{-16} \mathrm{~s}$, respectively, and values of the activation energy $E_{a}$ around $800 \mathrm{~K}$. These values for the attempt time are much lower than commonly observed in SPM systems $\left(10^{-9} \mathrm{~s}\right)$ and $\mathrm{SG}$ systems $\left(10^{-13} \mathrm{~s}\right)$. In addition, the values of $E_{a}$ around $800 \mathrm{~K}(69 \mathrm{meV})$, are much higher to that observed for the glassy peaks $(109 \mathrm{~K})$. Thus, it seems that the dynamics of these low temperature contributions could be ascribed to non-Arrhenius, fast relaxation processes. It has been shown for single domain particles, when the energy barrier to reverse the particle magnetization is small, the decay is found to be of non purely Arrhenius character [25-27]. According to these theoretical studies, fast relaxation processes are not consistent with the thermal activation over a single barrier with an Arrhenius exponential decay, or decay through parallel processes of Arrhenius type occurring at different rates, instead a more complex theoretical approach is needed [25]. It should be considered, for instance, a sequence of different metastable states between the initial and final states [26].

The existence of this low-temperature special relaxation can be connected also to an intricate spin arrangement, with a remarkable similarity to an amorphous arrangement. The particles are randomly placed with some of them behaving a single-domain particles; it is expected that some of them could be physically connected (allowing direct exchange coupling). Secondly, others could behave more independently, resembling a complex SPM with some interparticle (dipolar) interactions $[15,20]$. The combination of these two ingredients may result in the mentioned metastable states in the relaxation. As a matter of fact, the shape of the susceptibility curves and the interpretation shows a parallelism to that concluded for Fe-Zr and Fe-Zr-B amorphous alloys, with two different spin environments at different lengthscales resulting in a spin-disordered structure at low temperatures $[18,19,28]$.

It turns out that, in Re nanocrystalline materials, the modification of the physical properties due to the reduction of the size of the particles leading to a stronger influence of the surface effects is remarkable [29]. This can be further illustrated by the Arrott plots at different temperatures, shown in Figure 11, for the $40 \mathrm{~h}$ milled alloy. By contrast with those described above for the bulk sample, there is a change in the curvature between the low temperature region (Figure 11 a) and high temperature ones (Figure $11 \mathrm{~b}$ ). This situation resembles that observed in the mechanically milled $\mathrm{GdAl}_{2}$ alloy [30]. In addition, the overall behavior of these Arrott plots resemble those theoretically described for random anisotropy systems [31]. For the $\mathrm{GdAl}_{2}$ milled alloy, it was concluded that the disorderinduced random exchange and anisotropy within the grains and the boundaries leads to the freezing of FM regions at low temperatures into a Cluster Glass (CG) state [30]. This situation was equally observed in $\mathrm{TbAl}_{2}$ milled alloys [8], and it is bound to be the case in the Tb-Al-Fe milled samples as well. To end up, the described reduction of the coercivity with the decrease of the size of the particles should also be connected to the presence of a fraction of singledomain particles. It is well-known that there is an increase of the anisotropy with the decrease of size within the multidomain state. Eventually there is a particle size in which only single domain particles are energetically favorable in the alloy. In such a situation, there stands an effective random magnetic anisotropy which is averaged (reduced) by the number of particles and, consequently, a decrease of the coercivity. This decrease is very abrupt, scaling with $D^{-6}$ inside the single domain region [32].

\section{Conclusions}

Summarizing, the measurements of the magnetic properties in bulk $\mathrm{TbAl}_{1.5} \mathrm{Fe}_{0.5}$ alloy show the presence of a FM state which is modified at lower temperatures with the presence of a glassy behavior. This alloy shows a huge increase of the coercivity from $0.08 \mathrm{kOe}$ in bulk $\mathrm{TbAl}_{2}$ to 15 $\mathrm{kOe}$. In the milled samples (25 h and $40 \mathrm{~h}$ ), the coercivity decreases according to the presence of nanoparticles. In addition, static DC- and dynamic AC-magnetic susceptibility results indicate the presence of a glassy magnetic state in both bulk and nanometric $\mathrm{TbAl}_{1.5} \mathrm{Fe}_{0.5}$ alloys around $T_{0}$ $=62 \mathrm{~K}$. Moreover, below $T_{0}$, additional low frequency dependent peaks $T^{*}$, have been observed in the milled samples. These magnetic contributions cannot be described by a Vogel-Fulcher law, and parameters derived from an Arrhenius process yield uncommon values. Some studies attribute this to fast relaxation processes over small energy barriers. Naturally the intricate and disordered nature of the milled alloys not only in their local structure but in the particle arrangement favors the presence of such special spin relaxations. It would be interesting to extend this kind of study to other Re systems, especially what concerns to those low temperature contributions and to disclose in detail the magnetic structure via low temperature neutron and/or Mössbauer spectroscopy.

\section{Acknowledgements}

This work has been supported by the MAT 2008-06542C04 and MAT2011-27573-C04 projects.

\section{References}

[1] J.M.D Coey, Rare-Earth Iron Permanent Magnets (Monographs on the Physics and Chemistry of Materials), Oxford University Press, USA, 1996. 
[2] S.J. Collocott, J.B. Dunlop, H.C. Lovatt and V.S. Ramsden, Mat. Sc. Forum 315-317 (1999), p. 77.

[3] A.M. Gabay, N.G. Akdogan, M. Marinescu, J.F. Liu and G.C. Hadjipanayis, J. Phys.: Condens. Matter 22 (2010), p. 164213.

[4] J. Geshev, L. Bozukov, J.M.D. Coey, M. Mikhov, J. Magn. Magn. Mat 170 (1997), p. 219.

[5] E.M. Levin, V.K. Pecharsky and K.A. Jr, Gschneidner, J. Appl. Phys. 90 (2001), p. 6255.

[6] D.X. Chen, V. Skumryev and J.M.D. Coey, Phys. Rev. B 53 (1996), p. 15014.

[7] C. Piquer, J. Bartolome, C. de Francisco and J.M. Muñoz, Phys. Rev. B 79 (2009), p. 174430.

[8] D.P. Rojas, L. Fernández Barquín, J. Rodríguez Fernández, J.I. Espeso, J.C. Gómez Sal, J. Phys.: Condens. Matter 19 (2007), p.186214.

[9] D.P. Rojas, L. Fernández Barquín, J. Rodríguez Fernández, J.I. Espeso, J.C. Gómez Sal, J. Phys.: Conf. Series 200 (2010), p.072080.

[10] M.A. Laguna-Marco, C. Piquer and J. Chaboy, Phys. Rev. B 80 (2009), p. 144419.

[11] K. Williamson and W. H. Hall, Acta Metallogr. 1 (1953), p. 22.

[12] E. Ma, Prog. Mat. Science 50 (2005), p. 413.

[13] A. Arrott, Phys. Rev. 108 (1957), p. 1394.

[14] J.A. Mydosh, Spin Glasses: an Experimental Introduction (Taylor and Francis), London, 1993.

[15] D. Fiorani, L. Bessais and J.L. Dormann, J. Phys. C: Solid St. Phys. 21 (1988), p. 2015

[16] M. Reissner, W. Steiner, J.P. Kappler, P. Bauer and M.J. Besnus, J. Phys. F: Met. Phys. 14 (1984), p. 1249.

[17] L.C.J. Pereira, D.P. Rojas and J.C. Waerenborgh, Intermetallics 13 (2005), p. 61.

[18] S.N. Kaul, V.Siruguri and G. Chandra, Phys. Rev. B 45 (1992), p. 12343.

[19] R. García Calderón, L. Fernández Barquín, S. N. Kaul, J.C. Gómez Sal, Pedro Gorria, J. S. Pedersen, and R. K. Heenan Phys. Rev. B 71 (2005), p.134413.

[20] J. Alonso, M.L. Fdez-Gubieda, J.M. Barandiaran, A. Svalov, L. Fernández Barquín, D. Alba Venero and I. Orue, Phys. Rev. B 82 (2010), p. 054406.

[21] J.A. Chelvane, S. Kasiviswanathan, M.V. Rao, and G. Markandeyulu, Bull. Mater. Sci. 27 (2004), p. 169.

[22] Po-sen Wong, S. Von Molnar, T.T.M. Palstra, J.A. Mydosh, H. Yoshizawa, S.M. Shapiro, and A. Ito, Phys. Rev. Lett. 19 (1985), p. 2043.

[23] R.N. Bhowmika, R. Ranganathan, R. Nagarajan, J. Magn. Magn. Mat. 299 (2006), p. 327.

[24] R. Nigam, A.V. Pan, and S.X. Dou, Eur. Phys. J. B 74 (2010), p. 429.

[25] M. Lederman, S . Schultz, and M. Ozaki, Phys. Rev. Lett. 73 (1994), p. 1986

[26] Rodrigo Arias, H. Neal Bertram, J. Magn. Magn. Mat 171 (1997), p. 209.

[27] E.D. Boemer and H. Neal Bertram, IEEE Trans. Magn. 34 (1998), p. 1678.

[28] L. Fernández Barquín, J.C. Gómez Sal, P. Gorria, J.S. Garitaonandia and J.M. Barandiaran, Eur. Phys. J. B 35 (2003), p. 3.

[29] D. P. Rojas, L. Fernández Barquín, J. Rodríguez Fernández, L. Rodríguez Fernández and J. Gonzalez, Nanotechnology 21 (2011), p. 445702.

[30] P.M. Shand, C.C. Stark, D.S. Williams, M.A. Morales, T.M. Pekarek and D.L. Leslie-Pelecky, J. Appl. Phys. 97 (2005), p. $10 J 505$.

[31] A. Aharony and E. Pytte, Phys. Rev. Lett 45 (1980), p. 1583.

[32] G. Herzer, IEEE Trans. Magn. 26 (1990), p. 1397. 